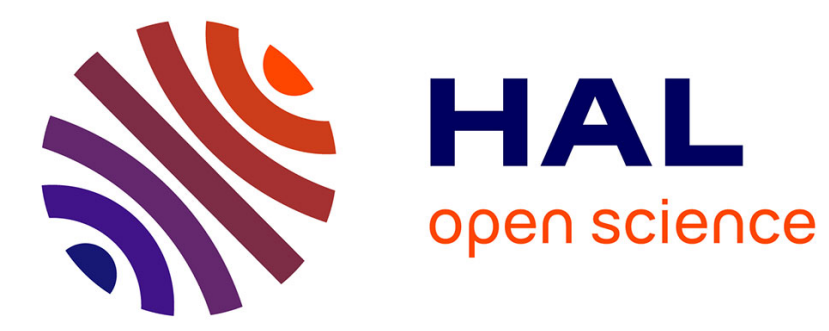

\title{
Uniaxial compression of silicon nanoparticles: an atomistic study on the shape and size effects
}

Dimitrios Kilymis, C Gérard, Jonathan Amodeo, Umesh V Waghmare, Laurent Pizzagalli

\section{- To cite this version:}

Dimitrios Kilymis, C Gérard, Jonathan Amodeo, Umesh V Waghmare, Laurent Pizzagalli. Uniaxial compression of silicon nanoparticles: an atomistic study on the shape and size effects. Acta Materialia, 2018, 158, pp.155-166. 10.1016/j.actamat.2018.07.063 . hal-02136329

\section{HAL Id: hal-02136329 \\ https://hal.science/hal-02136329}

Submitted on 22 May 2019

HAL is a multi-disciplinary open access archive for the deposit and dissemination of scientific research documents, whether they are published or not. The documents may come from teaching and research institutions in France or abroad, or from public or private research centers.
L'archive ouverte pluridisciplinaire HAL, est destinée au dépôt et à la diffusion de documents scientifiques de niveau recherche, publiés ou non, émanant des établissements d'enseignement et de recherche français ou étrangers, des laboratoires publics ou privés. 


\title{
Uniaxial compression of silicon nanoparticles: an atomistic study on the shape and size effects
}

\author{
D. Kilymis ${ }^{\mathrm{a}}$, C. Gérard ${ }^{\mathrm{a}}$, J. Amodeo ${ }^{\mathrm{b}}$, U. V. Waghmare ${ }^{\mathrm{c}}$, L. Pizzagalli ${ }^{\mathrm{a}, *}$ \\ ${ }^{a}$ Institut Pprime - CNRS UPR3346, Université de Poitiers - CNRS - ISAE-ENSMA, F86962 Chasseneuil Futuroscope Cedex, France \\ ${ }^{b}$ Université de Lyon, INSA-Lyon, MATEIS UMR5510 CNRS, 69621 Villeurbanne, France \\ ${ }^{c}$ Theoretical Sciences Unit, Jawaharlal Nehru Centre for Advanced Scientific Research (JNCASR), Bangalore 560064, India
}

\begin{abstract}
Molecular dynamics simulations were carried out to investigate the mechanical properties of silicon nanoparticles during uniaxial compression by a flat-punch indenter. We considered a large set of systems, with dimensions in the range $10 \mathrm{~nm}$ to $50 \mathrm{~nm}$, and various shapes like cubic (perfect and blunt), spherical, truncated spherical, and Wulff-shaped, as well as two compression orientations and two temperatures. Thorough analyses of the simulations first revealed that the relation between nanoparticle size and strength, usually termed as 'smaller is stronger', is critically dependent on the nanoparticle shape, at least for the investigated size range. For instance, a significant and size-dependent strength decrease is determined for facetted Wulff-like nanoparticles, but not for cubic or spherical systems for compression along $<100>$. We also found that the nanoparticle shape greatly influences plasticity. Several original plasticity mechanisms are obtained, among which the nucleation of half-loop V-shaped dislocation contained in two different $\{111\}$ planes, dislocations gliding in unusual $\{110\}$ planes, or the nucleation of partial dislocations in shuffle $\{111\}$ planes. Our investigations suggest that plasticity properties are mainly governed by the localization of shear stress build up during elastic loading, and the geometry of surfaces in contact with indenters, these two characteristics being intimately related to the nanoparticle shape.
\end{abstract}

Keywords: Nanoparticles; Mechanical properties; Plastic deformation; Silicon; Molecular dynamics simulations

\section{Introduction}

The mechanical properties of materials with nanoscale dimensions are often different from what we are used to with usual bulk systems. Maybe the most famous example is the seeming increase of strength associated to the reduction of dimensions, as observed in various materials [1, 2]. Several mechanisms such as dislocation nucleation from the surface or twinning were proposed to substantiate these observations [35]. They are related to the fact that at nanoscale, the surface becomes as important than the bulk, thus influencing both elastic and plastic properties.

It is noteworthy that most of the available results and the inferred conclusions were coming from investigations carried out on 1D (nanowires, nanopillars) and 2D (thin films) systems [610]. In fact, those are particularly well suited for nanomechanical testing, benefiting from the development of dedicated experiments following the pioneering work of Uchic et al. [11]. In comparison, our knowledge on the mechanical properties of OD systems like nanospheres or nanocubes is scarce [12]. This is rather unfortunate, since they constitute an important class of nanomaterials. In fact, nanoparticles are increasingly considered for potential applications in various domains, such as plasmonics, medicine, catalysis, and knowing the conditions under

\footnotetext{
${ }^{*}$ Corresponding author

Email address: laurent.pizzagalli@univ-poitiers.fr (L. Pizzagalli)
}

which they elastically deform or fail will allow to reduce the ageing degradation of functional properties. Understanding the mechanical response of nanoparticles is also critical in tribology and in applications where nanoparticles are envisaged as reinforcements in structural components. Furthermore, there is an intense research activity regarding the possible use of nanoparticles as anode-like materials in Li-ion batteries [13-15]. A successful future use of these systems is largely conditioned to a better understanding of their mechanical properties, and in particular to their propensity to fracture during lithiation [16].

Pioneering results on mechanical properties of nanoparticles were mainly published by the group of W.W. Gerberich, and essentially concerned silicon nanospheres $[17,18]$. Those studies reveal a rich and complex behavior, well different from what is known in bulk materials. For instance, it was shown that in small silicon nanoparticles stresses much higher than in the bulk could be achieved [19]. In certain conditions, these nanoparticles could be plastically deformed at room temperature, unlike bulk systems [20]. This feature was also observed in nanowires and nanopillars [7, 21-24]. Furthermore, experiments reveal that toughness is also enhanced in small silicon nanoparticles [25, 26].

Despite the aforementioned studies, which are the dominant plasticity mechanisms activated during the compression of silicon nanoparticles, still remains to be fully established. Several ones were proposed, such as amorphization [27], dislocation nucleation and propagation [28-31], and phase change [28, 30, 
32-34]. In the specific case of numerical simulations, it seems that the choice of the interatomic potential has a non negligible influence on the plastic deformation mode [28]. However, it is also highly likely that the activated mechanisms critically depend on parameters such as nanoparticles size, shape or orientation, which may explain the great diversity of reported behaviors.

In particular, the influence of the nanoparticle shape on mechanical properties is often overlooked compared to size dependence. A recent investigation revealing how the nanoparticle edge sharpness could influence the plastic response was performed by Amodeo and Lizoul [35], in the specific case of $\mathrm{Ni}_{3} \mathrm{Al}$ cubic nanoparticles. They show an unexpected transition from heterogeneous to homogeneous dislocation nucleation when the edges and corners of the nanoparticles become more rounded. Furthermore, they also find that the maximal attainable compressive stress greatly changes between sharp and rounded cubic systems, on a larger scale than size.

Despite the importance of these findings, there is a critical lack of investigations on this particular issue. Especially, it would be highly beneficial for our understanding to get further information on the relation between shape and plasticity, for as many as possible nanoparticle shapes, and more importantly acquired in a unique framework. This constitutes the motivation of the present work. We realized a comprehensive study of the mechanical response to uniaxial compression of silicon nanoparticles with several different shapes. Silicon is particularly appropriate for such investigations, because various nanoparticle shapes are stable or can be obtained by local quenching [36, 37]. Silicon is also characterized by a great variety of plasticity mechanisms [30, 38-42], whose are expected to depend on the shape too.

We used molecular dynamics, a method able to handle system sizes comparable to those reported in experiments, while allowing for a detailed atomistic analysis of plasticity mechanisms. A large set of nanoparticles with various shapes and sizes were tested, revealing that the nanoparticle shape is unquestionably a more important parameter than its size for the investigated size range. In fact, the nanoparticle shape is shown to largely influence the nanoparticle strength and plasticity mechanisms, as well as the existence of a size effect. This study also show the triggering of several original plasticity mechanisms, some of which being recently proposed in the literature.

\section{Methods}

Initial nanoparticle structures are prepared by carving selected shapes from a silicon lattice of lattice constant $a_{0}$ equal to $0.543 \mathrm{~nm}$ (Fig. 1). The chosen geometries, spherical, Wulfflike, cubic and rounded cubic, were all shown to be thermodynamically stable [36] and/or experimentally achievable [30]. Selected system sizes, defined as the distance between the topmost and bottommost atoms along the $<100>$ or $<111>$ orientations, range approximately from 10 to $50 \mathrm{~nm}$ (Table 1). The Wulff-like shaped nanoparticles are generated using the $\{111\}$, $\{100\}$ and $\{110\}$ surface energies calculated by Stekolnikov et al. [43], resulting in a truncated octahedron geometry. In the
Table 1: Initial dimensions of the different studied systems.

\begin{tabular}{cc}
\hline \hline Geometry & Dimensions $(\mathrm{nm})$ \\
\hline Sphere & $10.9 / 21.5 / 32.6 / 43.4 / 54.3$ \\
Wulff-like & $10.9 / 21.8 / 32.7 / 43.7 / 54.6$ \\
Cube $(\alpha=0.00)$ & $10.1 / 20.1 / 30.0 / 40.3 / 50.2$ \\
Cube $(\alpha=0.05)$ & $10.1 / 19.8 / 29.6 / 39.9 / 49.7$ \\
Cube $(\alpha=0.15)$ & $10.1 / 19.8 / 29.6 / 39.9 / 49.7$ \\
Cube $(\alpha=0.30)$ & $10.1 / 19.8 / 29.6 / 39.9 / 49.7$ \\
T-sphere- $n$ & $48.8(n=20) / 43.5(40) / 32.5(80)$ \\
& $<111>$ \\
Sphere & $10.8 / 21.4 / 32.4 / 43.4 / 54.1$ \\
Wulff-like & $10.8 / 21.5 / 32.5 / 43.5 / 54.2$ \\
\hline \hline
\end{tabular}

case of the perfect cubic nanoparticles, all single-coordinated atoms located on the edges are removed. These structures are next used for preparing blunt geometries with rounded corners and edges, following the procedure depicted by Amodeo and Lizoul [35]. Three geometries with blunting parameter $\alpha=0.05,0.15$, and 0.30 , are considered in this study for each cube size. Finally, we also prepare three additional configurations with a truncated-sphere shape by removing layers of atoms from the top and bottom of a $50 \mathrm{~nm}$ diameter sphere. In the following, these systems are called T-sphere- $n, n$ being the number of removed layers on each side.

An important aspect of molecular dynamics calculations is the selection of the interatomic potential. In the literature, most of the numerical simulations dedicated to mechanical properties of silicon nanostructures either used the Stillinger-Weber [44] or the Tersoff potential [45]. We know from previous works that neither of the two can provide a perfect description of the plastic deformation of silicon [46]. The main advantage of the Tersoff potential is its ability to reproduce the cubic diamond- $\beta$-tin transition under pressure $[32,33]$. However, it also tends to overestimate bonds strength, thus limiting dislocation nucleation and propagation unless for very high stresses and temperatures [47]. Conversely, the Stillinger-Weber (SW) potential is more appropriate for modelling dislocation mediated plasticity $[29,46]$, but it largely overestimates the pressure threshold for the $\beta$-tin phase transition. In this study, we chose the latter to model the silicon nanoparticles, but we use a recent reparametrization which improves several aspects like plasticity, amorphization, shear-transformation, over the original one [48]. Note that with this potential, the cubic diamond$\beta$-tin phase transition remains overestimated. This issue will be discussed later in the paper.

The molecular dynamics simulations are carried out using the LAMMPS package $[49,50]$. The Verlet algorithm with a timestep of 1 fs combined with the Nose-Hoover thermostat is used for the integration of equations of motion, within a constant volume-constant temperature thermodynamic ensem- 

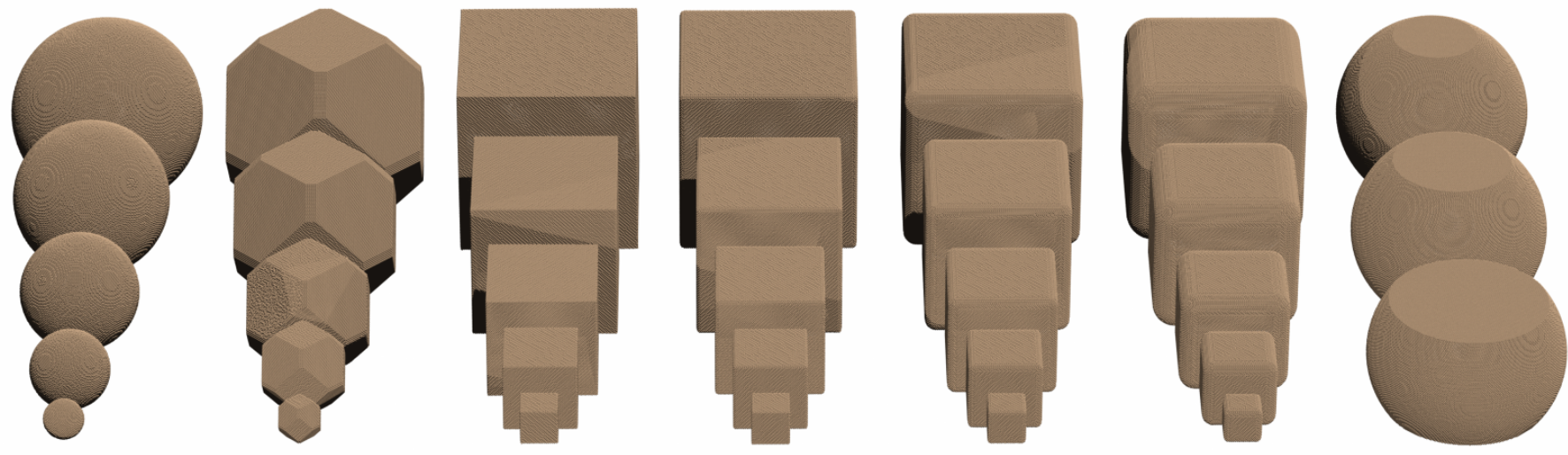

Figure 1: Overview of all silicon nanoparticles investigated in this work: spherical, Wulff-like, cubic (perfect), cubic $(\alpha=0.05)$, cubic $(\alpha=0.15)$, cubic $(\alpha=0.30)$, truncated spherical, from left to right. Except for the latter, five different sizes, reported in Table 1, were considered. The truncated-sphere nanoparticles are built by removing top and bottom layers from the largest sphere.

ble. All configurations are located in a computational box large enough to avoid interactions with periodic replicas. Prior to compression, each system is heated and equilibrated at $300 \mathrm{~K}$, allowing for surface reconstructions. Uniaxial compressions are performed at two different temperatures, $5 \mathrm{~K}$ and $300 \mathrm{~K}$, for all shapes and sizes, by initially placing infinite planar indenters, normal to the compression axis, on each side of the nanoparticles. As implemented in LAMMPS, the forces between these indenters and the nanoparticle are quadratic and purely repulsive, and proportional to a parameter $k$. In order to mimic an indenter much harder than the studied nanoparticles, $k$ is set to $1000 \mathrm{eV} \AA^{-3}$ in this work. The compression test is realized by moving both indenters towards each other at a constant velocity equal to $0.1 \AA / p s$. This leads to engineering strain rates roughly ranging between $10^{8}$ and $10^{9} \mathrm{~s}^{-1}$, which is typical of molecular dynamics calculations. We performed additional simulations for specific systems with a strain rate lowered by one order of magnitude. These tests reveal no noticeable qualitative and quantitative differences with the previous simulations. We also investigated the influence of surface reconstruction, by carrying out $5 \mathrm{~K}$ compression tests of nanoparticles with unreconstructed surfaces, obtained by skipping the initial $300 \mathrm{~K}$ annealing stage. The comparison with the standard case shows that surfaces reconstruction also have no noticeable effect on the elastic modulus, the yield stress and strain, as well as plastic deformation mechanisms.

Most of the previously published works were concerning uniaxial compressions along $<100>$ and $<111>$ orientations. For a meaningful comparison, all configurations were compressed along $<100>$ in this study. For the $<111>$ orientation, only spherical and Wulff-like nanoparticles were considered. In that case, the systems are initially rotated in order to align $\{111\}$ faces with the indenters.

To determine the true compression stress, it is necessary to estimate the contact surface area between the indenter and the nanoparticle. Note that the notion of contact at the nanoscale is not clearly defined [51]. In this study, we consider that an atom is in contact with the indenter if the separation is lower than $1 \AA$.
Reasonable increase or decrease of this threshold lead to negligible differences in stress-strain curves. Knowing the atoms in contact, the associated surface area can then be determined by Delaunay triangulation and the true stress calculated as the ratio between the force acting on one indenter divided by the surface value. Other approaches were proposed to determine the contact surface $[51,52]$, and we performed different analyses to check how this could influence our results. We found that the Delaunay triangulation is probably the best one to investigate shape effects, because no particular contact geometry is assumed. However, irrealistic values can be obtained in the very first deformation stages that include few atoms in contact. This issue has no consequences on the results presented in the following.

Atomic stresses for each atom $i$, having mass $m_{i}$ were calculated using the Virial theorem:

$$
\sigma_{\alpha \beta}^{i}=-\frac{1}{V_{i}}\left[\frac{1}{2} \sum_{j=1}^{N} x_{\alpha}^{j} f_{\beta}^{i j}+\mathrm{m}^{i} v_{\alpha}^{i} v_{\beta}^{i}\right]
$$

where $x_{\alpha}^{j}, v_{\alpha}^{j}$ are the positions and velocities of all neighboring atoms along the $\alpha$ direction, while $f_{\beta}^{i j}$ is the force they exert on the atom $i$ (of mass $m_{i}$ ) along the $\beta$ direction. The atomic volumes $V_{i}$ were calculated using the Voronoi tessellation method. Since the latter quantity is ill defined for surface atoms, atomic stresses are used only for non-surface atoms. We also compute the von Mises stresses defined as

$$
\begin{aligned}
\sigma_{v m}= & \frac{1}{\sqrt{2}}\left[\left(\sigma_{x x}-\sigma_{y y}\right)^{2}+\left(\sigma_{y y}-\sigma_{z z}\right)^{2}+\left(\sigma_{z z}-\sigma_{x x}\right)^{2}\right. \\
& \left.+6\left(\sigma_{x y}^{2}+\sigma_{x z}^{2}+\sigma_{y z}^{2}\right)\right]^{1 / 2}
\end{aligned}
$$

Post-processing analyses and visualization for identifying specific configurations and dislocations were performed using the visualization and analysis software Ovito [53, 54]. 


\section{Results}

\subsection{Mechanical response of Si nanoparticles}

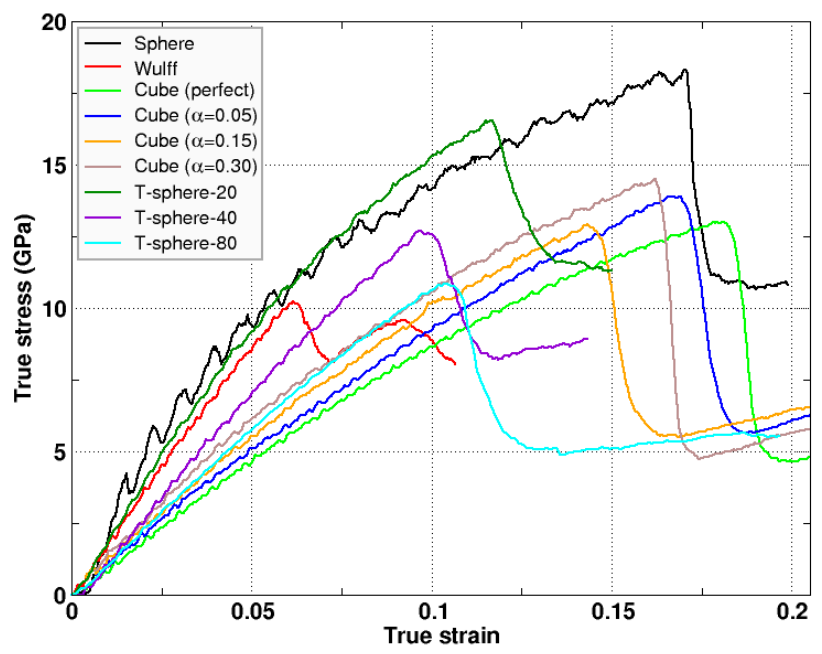

Figure 2: Compressive true stress versus true strain, for nanoparticles of sizes around $50 \mathrm{~nm}$ with various shapes, compressed along $<100>$ at $5 \mathrm{~K}$.

We first examine the mechanical response of the compressed nanoparticles through stress-strain curves. In this section, we focus on results obtained at $5 \mathrm{~K}$, since they do not exhibit significant differences with $300 \mathrm{~K}$ simulations. As an example, we consider the case of $50 \mathrm{~nm}$ nanoparticles of various shapes compressed along $\langle 100\rangle$. The overall shapes of stress-strain curves, represented in the Figure 2, appear to be relatively close. The stress increases linearly at the very beginning of the compression, but with a clear deviation from linearity as soon as the strain exceeds few percents. Smooth stress fluctuations are observed, except for the sphere, for which ripples can be seen. The latter are due to surface contact variations associated with the layer by layer flattening of the contact surface below the indenter, as will be shown in the next sections. Then, the stress reaches a maximum value, which defines the yield point. It corresponds to the highest stress that the nanoparticle can sustain before yielding. Beyond this point, a significant stress reduction is observed, suggesting that plastic deformation was initiated.

Focusing on the initial deformation stage, one can extract elastic moduli from each curve. Assuming linearity from $0 \%$ to $2 \%$, values between $96 \mathrm{GPa}$ (perfect cube) and $296 \mathrm{GPa}$ (sphere) are calculated. Note that only in the case of the perfect cube this value is equivalent to the Young modulus as classically defined. For the modified SW potential used in this work, the bulk $<100>$ Young modulus is equal to $102 \mathrm{GPa}$, in excellent agreement with the computed $96 \mathrm{GPa}$.

Deviations from linearity at higher strains are more or less pronounced depending on the case. For one part, stress is partially relaxed by surfaces during loading. In addition, deviations could also be correlated to contact surface variations. Hence the lowest ones correspond to geometries for which the contact surface area show little or no variations during loading, while the largest deviation is obtained for the sphere.

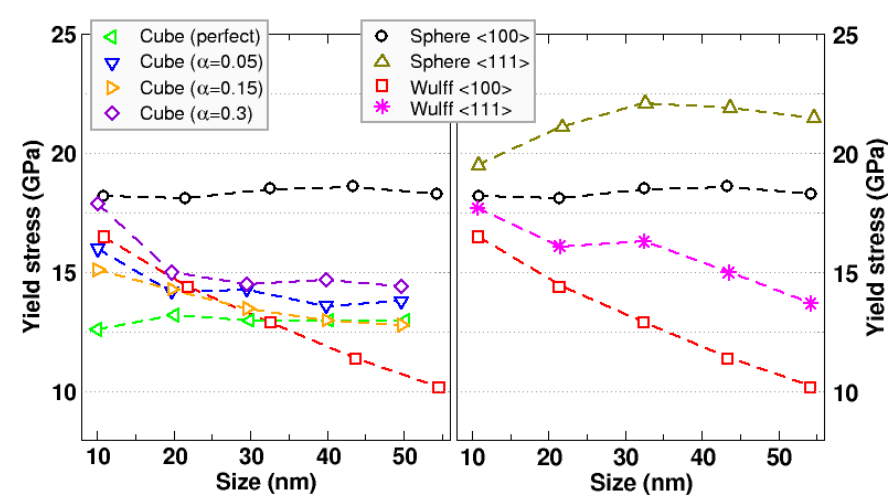

Figure 3: Yield stress as a function of size, at $5 \mathrm{~K}$. Results for compressions along $<100>$ are shown on the left graph, and those for spherical and Wulff-like nanoparticles and both $<100>$ and $<111>$ orientations are represented on the right graph. Dashed lines have been added between data points for visualizing trends.

As seen in Fig. 2, yield stress values range from 10.2 GPa for the Wulff-like to $18.3 \mathrm{GPa}$ for the sphere, i.e. a maximum stress difference of $8.1 \mathrm{GPa}$ between nanoparticles of similar dimensions. Such a large shape effect on stress is in agreement with recent findings [35]. Nevertheless, there is no evident relation between shape and yield stress. At first sight, one could argue that high yield stresses are related to low contact surface areas. This would explain the maximum stress for the perfect sphere, and decreasing values when an increasing number of layers are removed in T-spheres (thus increasing the contact surface area). However it does not explain why the yield stress for the Wulfflike nanoparticle is lower than for the cubic one, nor why the yield stress does not monotonically increase as a function of $\alpha$.

Increasing the temperature to $300 \mathrm{~K}$ leads to yield stress reduction of about $0-2 \mathrm{GPa}$, depending on the system. Wagner et al. reported yield stress values of about $10 \mathrm{GPa}$ for $50 \mathrm{~nm}$ blunted cubic silicon nanoparticles, uniaxially compressed at RT along $<100>$ [30]. In our $300 \mathrm{~K}$ calculations, yield stress values ranging between 11.8 and $13.6 \mathrm{GPa}$ are determined for cubic nanoparticles with $\alpha$ in the range $0-0.3$, thus in fair agreement with experiments.

Figure 3 shows the yield stresses extracted from 5 K stressstrain curves, as a function of the nanoparticle size, for different shapes and compression orientations. Focusing first on the $<100>$ compressed Wulff-like nanoparticle, we observe a marked increase of the yield stress associated to size reduction, from 10.2 GPa to 16.5 GPa for the smallest nanoparticles. A similar increase is also obtained for the $<111>$ orientation, although the increase rate is lower (from 13.7 GPa to $17.7 \mathrm{GPa}$ ). The yield stress is also systematically higher in this orientation compared to $<100>$. To our knowledge, the mechanical properties of silicon Wulff shaped nanoparticles were not investigated yet, and therefore there are no data to compare with. Nevertheless, a recent study of the $<111>$ compression of Wulff shaped FCC nanoparticles hints that the relation between yield stress and size should be a power law, with an exponent of 0.5 [55]. The authors also proposed that this exponent solely depends on the nanoparticle geometry, i.e. more precisely on the angles be- 
tween $\{111\}$ and $\{100\}$ planes, and not on material properties. Since Wulff shapes of FCC and silicon nanoparticles are similar, it is interesting to examine whether such a relation could be relevant here. We find that our data could be approximately fitted by the expression $\sigma_{y}=24 d^{-0.13}$, thus with a very different exponent than in [55]. One possible explanation is that plasticity mechanisms are different in silicon and in FCC metals, as will be shown in the next sections.

The spherical nanoparticle is characterized by the highest yield strength values, with maxima of $18.6 \mathrm{GPa}(22.1 \mathrm{GPa})$ for the $<100>(<111>)$ orientation, respectively. There is no marked variation associated to the nanoparticle size for the $<100>$ orientation. For $<111>$, the yield strength tends to first increase with size, reaches a maximum value for the midsize configuration, then slightly decreases for the largest diameter. Overall, these values are in qualitative agreement with past theoretical investigations. Using the original SW potential, Chrobak and co-workers recently reported yield strength values in the range $21-23.5 \mathrm{GPa}$ for 5-10 $\mathrm{nm}$ diameter spherical nanoparticles compressed along $<100>$ [56]. A much lower value of about $2 \mathrm{GPa}$ was also proposed for $40 \mathrm{~nm}$ nanoparticles [28], but the authors used a completely different yield stress definition than in most published works, preventing a meaningful comparison. Further data are available from Tersoff potential calculations, also showing larger yield stresses for compression along $<111>$ compared to $<100>[33,34]$. A quantitative comparison remains difficult, since largely different yield stress values are reported despite using the same potential [32-34]. Also, one should keep in mind that the activated plasticity mechanisms are different with this potential, as will be presented in the next section. This may also explain why our results disagree with the Tersoff-based calculations by Hong et al. [34], which suggested a large stress reduction when the nanosphere diameter increases from $10 \mathrm{~nm}$ to $50 \mathrm{~nm}$ for the $\langle 100\rangle$, whereas no significant variations are found in the present work.

Similarly, no size effect on yield stress is obtained in the case of the perfect cube, with values ranging from 12.6 to $13.2 \mathrm{GPa}$ (Fig. 3). However, smoothing the cube edges and corners leads to higher stresses for the smallest nanoparticles. This effect tends to vanish for system sizes greater or equal to $30 \mathrm{~nm}$. Recent experimental investigations of $<100>$ compressed cubic silicon nanoparticles also revealed a weak size dependence for sizes from 20 to $65 \mathrm{~nm}$, and yield stress values between 9 and $12 \mathrm{GPa}$ [30]. These nanoparticles are characterized by blunt edges, and should be compared to our models with $\alpha$ equal to 0.15 or 0.3 . For those, a negligible size dependence is also found for sizes like in experiments (Fig. 3), although our computed yield stress values are larger, in the range 13-15 GPa. As already mentioned, such a difference could be due to the large difference in strain rates between experiments and calculations.

\subsection{Plasticity mechanisms}

\subsubsection{Wulff-like shape $-<100>$ orientation}

The onset of plasticity in Wulff-like nanoparticles occurs through the heterogeneous nucleation of dislocations from the a)

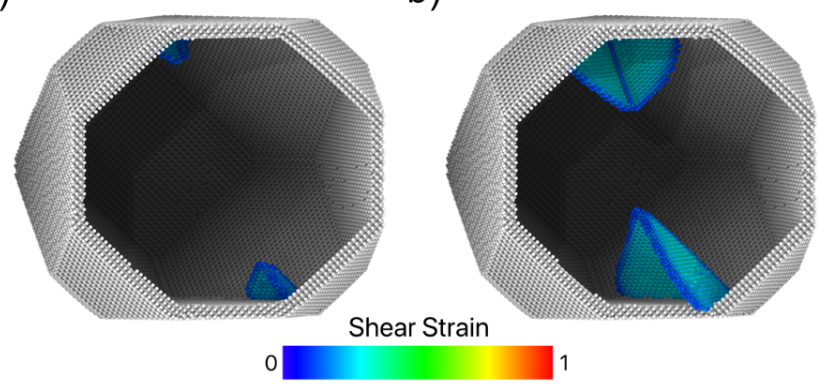

C)

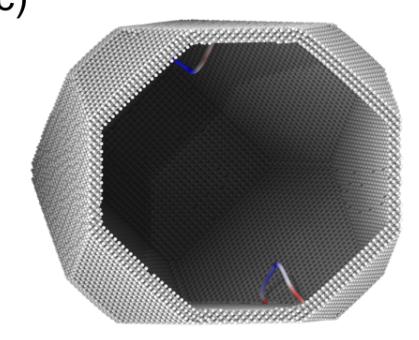

d)

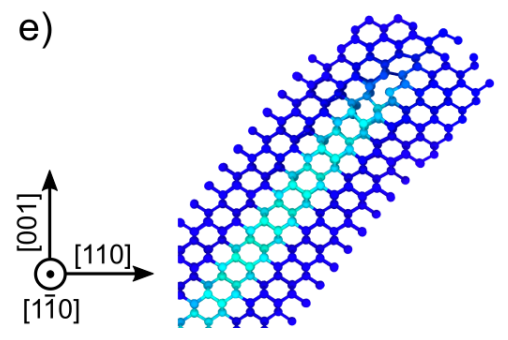

Figure 4: Onset of plastic deformation at $5 \mathrm{~K}$ in a $20 \mathrm{~nm}$ Wulff shaped silicon nanoparticle uniaxially compressed along $<100>$. For clarity, perfect crystal atoms inside the nanoparticle are not shown, and the nanoparticle was cut. von Mises shear strain visualization at true strains equal to 0.099 (a) and to 0.102 (b). (c-d) Dislocation line visualization using the dislocation extraction algorithm included in Ovito for the same respective strains than (a-b). Red (blue) colored segments correspond to the screw (edge) orientation. (e) Slice of the configuration shown in (b), revealing the dislocation core configuration.

contact surfaces (Fig. 4). The Burgers vector of these dislocations is $\left(a_{0} / 2\right)<110>$, and they are located in shuffle $\{111\}$ planes, as expected for the plastic deformation of silicon at low temperature (i.e. below the brittle to ductile transition temperature) [38]. However a rather unusual feature of these half loop dislocations is the fact that they glide in two different $\{111\}$ planes. Following nucleation, the half-loop expands in both $\{111\}$ planes, with a leading edge at their intersection (Fig. 4$\mathrm{b}, \mathrm{d})$. Each arm of the dislocation is composed of a moving front with a $60^{\circ}$ character, connected to the surface by a screw segment. Well grown half-loops show a recognizable V-shape. No noticeable effect of temperature or size is found. In particular, in all simulations save one, the plastic deformation proceeds almost simultaneously from both top and bottom surfaces, with a 0.002 maximum strain difference between events.

Hale and co-workers reported such V-shaped halfloop dislocations for the uniaxial compression of silicon nanospheres [29]. In this study, the plastic deformation was initiated by the homogeneous nucleation of a dislocation loop on a $\{110\}$ plane, followed by propagation on connecting $\{111\}$ 
planes by cross-slip. In the present work, the nucleation is heterogeneous, and occurs in a $\{111\}$ plane in the vicinity of the corner connecting the $\{100\}$ contact surface, and lateral tilted $\{111\}$ facets. For the largest nanoparticles, a very thin $\{110\}$ facet is separating the two $\{111\}$ facets, bringing some similarities with the previous study. However, the same mechanism occurs for small nanoparticles, for which the $\{110\}$ facet is no larger than one atom. It is also interesting that similar V-shaped dislocations were reported in literature, albeit in a different context. In fact, recent investigations of the formation of misfit edge dislocations in a strained $\mathrm{GeSi}$ thin film on $\mathrm{Si}(100)$ suggested that a mechanism involving V-shaped dislocations (called split dislocation in this work) would be energetically favored compared to the commonly assumed half-loop dislocation [57].

We also analyze the fine structure of the dislocation cores, in particular for the $60^{\circ}$ segment, since several possible core configurations were reported in the literature [58]. Figure 4e shows the core of a $60^{\circ}$ dislocation segment, extracted from large scale simulations. One can unambiguously recognize the mobile S1 core originally proposed by Hornstra [59]. Although previously reported as no stable [58], recent first-principles calculations showed that in presence of large tensile or compressive strains, its stability is greatly improved [60].

\subsubsection{Wulff-like shape $-<111>$ orientation}

a)

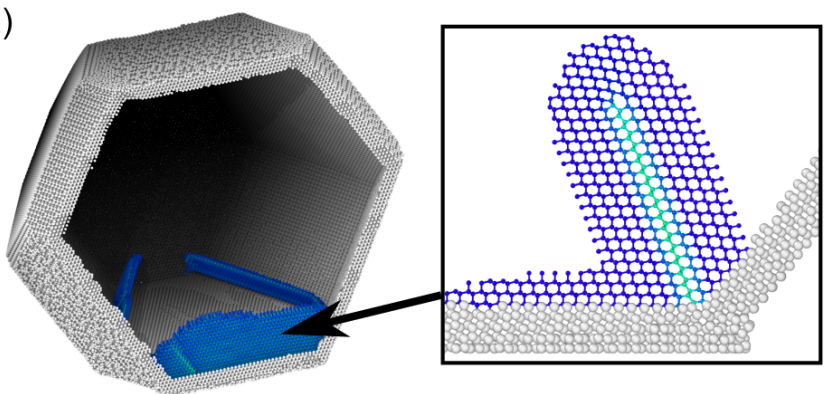

b)

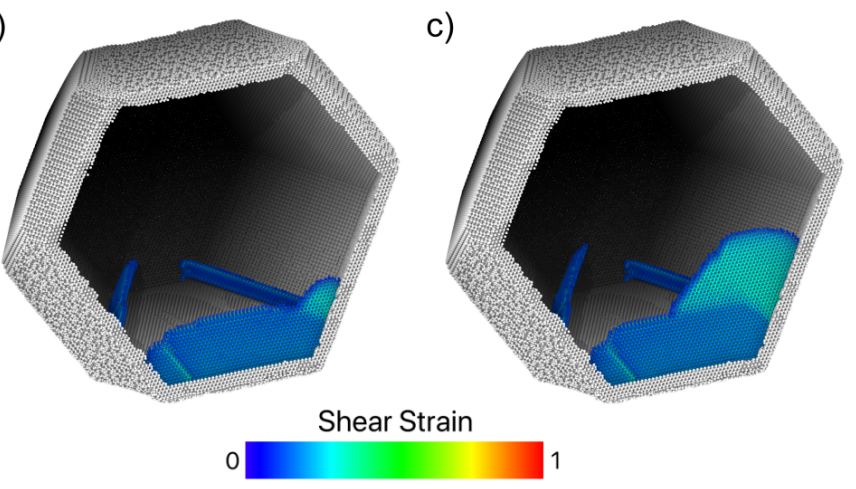

Figure 5: Onset of plastic deformation at $5 \mathrm{~K}$ in a $32.5 \mathrm{~nm}$ Wulff shaped silicon nanoparticle uniaxially compressed along $<111>$. For clarity, perfect crystal atoms inside the nanoparticle are not shown, and the nanoparticle was cut. von Mises shear strain visualization at true strains equal to 0.108 (a), to 0.110 (b), and to 0.111 (c). In the upper right, a slice view of the configuration shown in (a) reveals the disordered surface edge, the dislocation core configuration, and the stacking fault left behind.

The analysis of plastic deformation reveals a completely dif- ferent scenario when the Wulff shaped nanoparticles are compressed along the $<111>$ orientation instead. First, appreciable changes of the atomic structure are observed during the apparent elastic loading of the nanoparticle. It starts with a spatially limited disordering of the atoms forming the corners of the contact surface (see the enlarged region of Fig. 5-a). At strains about 0.076 one can see slip events initiated from the edges formed at the intersection of the contact surface and $\{100\}$ lateral facets. Each one corresponds to the formation and propagation of a dislocation, leaving behind a stacking fault in a glide set $\{111\}$ plane. From the analysis of atomic displacements, a Burgers vector $\left(a_{0} / 6\right)<112>$ is determined. However, the core structure, shown in the Fig. 5, is characterized by 7 and 5-fold rings, and does not resemble known partial dislocation cores [61]. It corresponds to a shuffle set $90^{\circ}$ partial dislocation core, which was proposed in pioneering works [62]. Such dislocations were already identified in molecular dynamics simulations [63], and recent experiments also support their existences [42]. The dislocation propagation is very slow, and only small areas are swept for strains in the range 0.076-0.110 (Fig. 5). This only induces a weak softening in the stress-strain curve. The elastic limit equal to 0.110 corresponds to the nucleation of a perfect $60^{\circ}$ dislocation from the $90^{\circ}$ partial core, gliding in a $\{111\}$ shuffle plane and leaving behind a $30^{\circ}$ partial dislocation. The perfect dislocation then expands through the nanoparticle very rapidly. The plastic deformation is then mainly due to nucleation and propagation of additional perfect $60^{\circ}$ dislocations.

We find no significant influence of size or temperature on the whole process. Depending on the case, only one or both contact surfaces are concerned by the initiation of plasticity.

\subsubsection{Spherical shape $-<100>$ orientation}

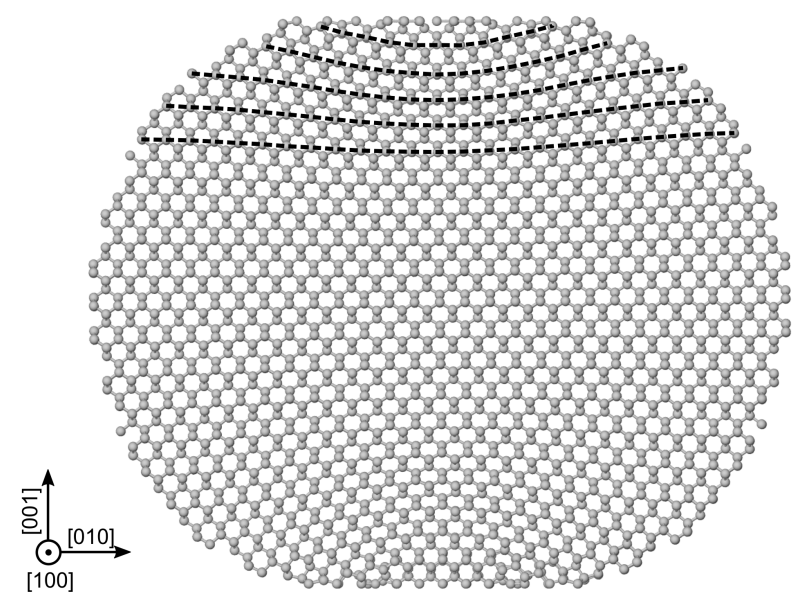

Figure 6: Cross section view of a $10.9 \mathrm{~nm}$ spherical nanoparticles compressed along $\langle 100\rangle$, just before the elastic limit is reached. The large distortion of the atomic layers close to the top indenter is emphasized by dashed thick lines.

The $<100>$ compression of nanoparticles with a spherical shape is remarkably different from the $<100>$ Wulff shape case. In fact, the elastic regime is now ongoing until much larger strains, and is characterized by a significant and pro- 
a)

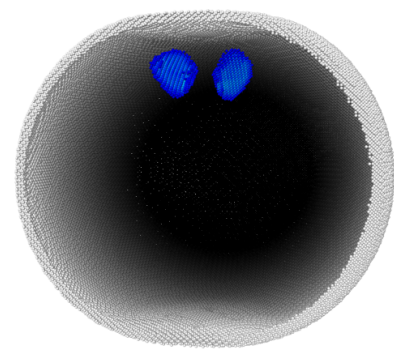

c)

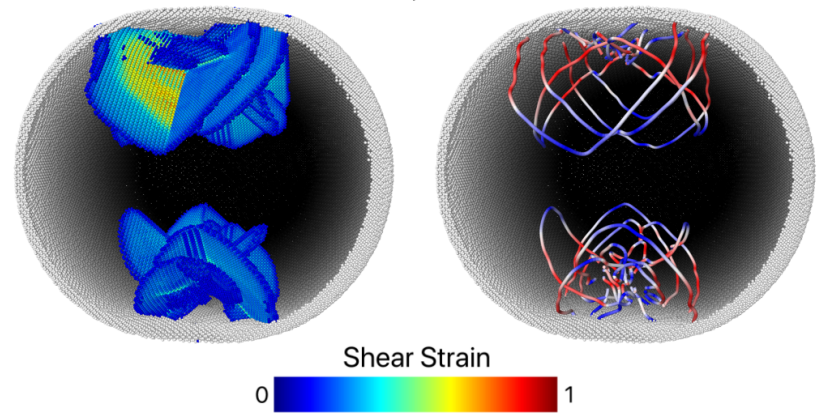

Figure 7: Onset of plastic deformation at $5 \mathrm{~K}$ in a $32.6 \mathrm{~nm}$ spherical silicon nanoparticle uniaxially compressed along $<100\rangle$. For clarity, perfect crystal atoms inside the nanoparticle are not shown, and the nanoparticle was cut. (a-c) von Mises shear strain visualization at true strains equal to 0.1810 (a), to 0.1815 (b), and to 0.1823 (c). (d) Dislocation line visualization using the dislocation extraction algorithm included in Ovito for the same strain than (c). Red (blue) colored segments correspond to the screw (edge) orientation.

gressive bending of the $\{100\}$ atomic layers, the deformation being larger in the vicinity of the indenters (Fig. 6). This process appears similar to the indentation of the nanoparticle by the topmost (and bottommost) atomic layers, and is associated with atomic rearrangements on surfaces leading to ripples in the stress-strain curve (Fig. 2). Compared to previous works, no phase transformation is observed, and a spherical geometry is recovered if indenters are pulled back before the yield point.

Nanoparticle yielding occurs by the homogeneous nucleation of $\{111\}$ shuffle dislocation loops with $\left(a_{0} / 2\right)<110>$ Burgers vector below (above) the top (bottom) indenters, in weakly deformed regions (Fig. 7-a). These loops expand until they meet, which induces cross-slip of the screw segments in another $\{111\}$ slip systems, and the formation of V-shaped dislocations (Fig. 7-b). In few cases, it seems that V-shaped dislocations are formed from the very beginning of plastic deformation. Also, we find that the connection between the two $\{111\}$ slip planes in V-shaped dislocations is either atomically sharp, or could be constituted of a small area belonging to a $\{110\}$ plane, as in [29]. This process repeats as deformation continues, generating new dislocations in all four possible $\{111\}$ glide systems (Fig. 7-c,d). No significant influence of size or temperature is observed.

\subsubsection{Spherical shape $-<111>$ orientation}

Considering now the compression along $<111>$, we find out that the formation of the localized elastically deformed phase is less pronounced. The elastic deformation of the nanoparticle is apparently more homoegeneous during the elastic regime. a) b)

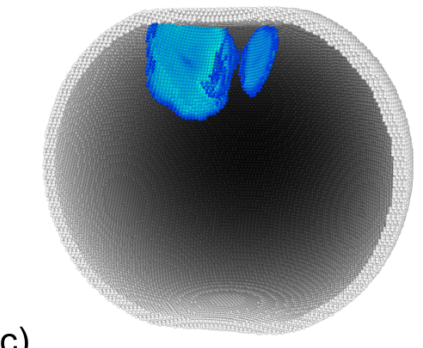

c)

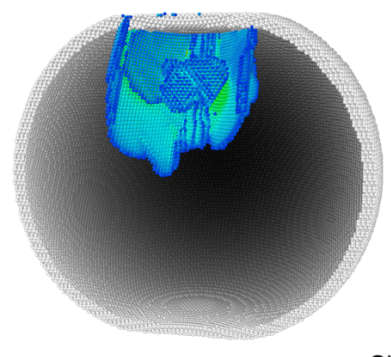

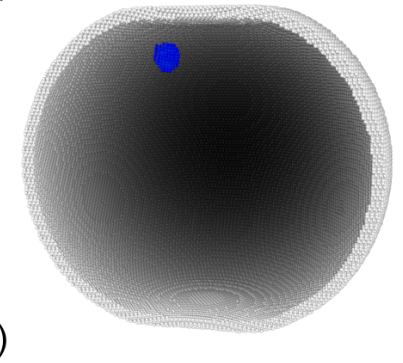

d)

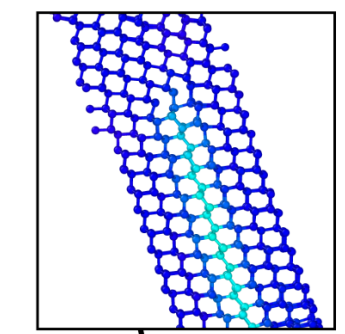

e)
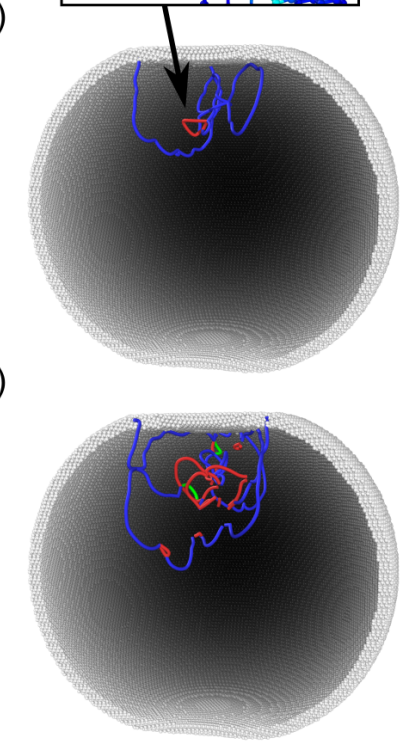

Shear Strain
Figure 8: Onset of plastic deformation at $5 \mathrm{~K}$ in a $32.6 \mathrm{~nm}$ spherical silicon nanoparticle uniaxially compressed along $<111>$. For clarity, perfect crystal atoms inside the nanoparticle are not shown, and the nanoparticle was cut. (ac) von Mises shear strain visualization at true strains equal to 0.157 (a), to 0.159 (b), and to 0.160 (c). (d-e) Dislocation line visualization using the dislocation extraction algorithm included in Ovito for the same strain than (c-d). The blue color corresponds to $\left(a_{0} / 2\right)<110>$ dislocations, whereas red and green colors are attributed to different dislocations, not formally identified by Ovito.

This is in agreement with the fact that the $<111>$ direction in silicon corresponds to the highest strength. The elastic limit corresponds to the homogeneous nucleation of a perfect dislocation loop, in a region close to one indenter (Fig. 8-a). The dislocation is characterized by a $\left(a_{0} / 2\right)<110>$ Burgers vector, and glide in shuffle $\{111\}$ planes. The expansion of this primary dislocation is accompanied by the nucleation of other similar dislocations, and propagation in other $\{111\}$ planes by crossslip for instance.

However, we detect additional unusual events during the expansion/nucleation of these perfect dislocations. In fact, at several places, the $60^{\circ}$ dislocation core dissociates, leading to the formation of dislocation loops in intersecting $\{111\}$ planes (Fig. 8-b,d). These dislocations are actually partial dislocations, with a Burgers vector equal to $\left(a_{0} / 6\right)<112>$. The core represented in Figure 8-d is equivalent to the one depicted in the Figure 5-a, although their structure might appear to differ at first sight, because snapshots correspond to different steps of their migration. A glide set $\{111\}$ stacking fault is growing when the 
loop expands, and a sessile partial core remains at the original location of the $60^{\circ}$ dislocation segment. As usual, we do not observe a significant influence of temperature or size on the results.

\subsubsection{Truncated spherical shape}

a)

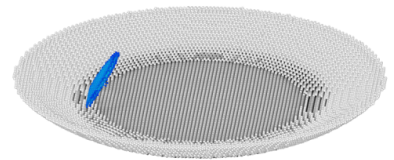

c)

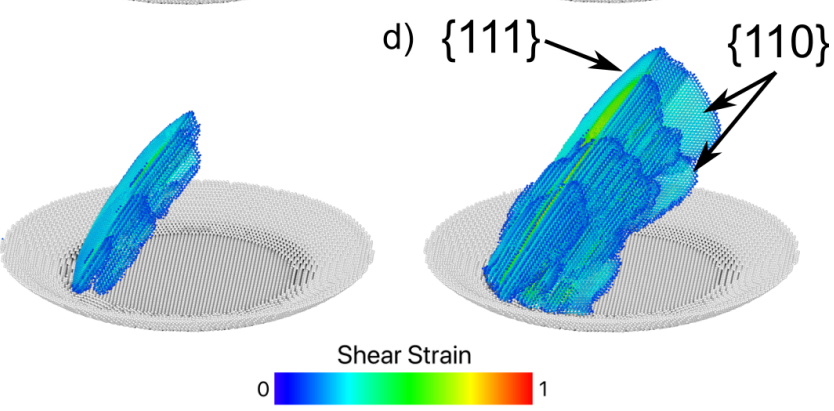

Figure 9: Onset of plastic deformation at $5 \mathrm{~K}$ in a $50 \mathrm{~nm}$ T-sphere-20 silicon nanoparticle (20 atomic layers removed on both top and bottom $\{100\}$ surfaces). For clarity, only atoms in a small part of the nanoparticle are shown. (a-d) von Mises shear strain visualization at true strains equal to 0.115 (a), 0.117 (b), 0.118 (c), 0.119 (d).

The analysis of the compression along $<100>$ of truncated spherical nanoparticles is particularly instructive, although such shapes are probably not experimentally feasible. As shown in Fig. 9, plastic deformation starts by the nucleation of half loop dislocations in $\{111\}$ planes, at the edge of the circular contact surface corresponding to the truncated region. These dislocations are perfect shuffle dislocations with a Burgers vector equal to $\left(a_{0} / 2\right)<110>$. Their expansion is driven by (i) the $60^{\circ}$ front segment gliding towards the nanoparticle interior (ii) the expansion of the screw segments, attached to the contact edge, and cross-slip in $\{110\}$ planes (Fig. 9-b-c). This mechanism allows for the plastic deformation to follow the circular shape of the contact surface (Fig. 9-d). Compared to Wulff-like nanoparticles, the absence of contact surface edge corners prevents the formation of $\mathrm{V}$-shaped dislocations.

A similar mechanical response is obtained for all T-sphere- $n$ configurations, and for both $5 \mathrm{~K}$ and $300 \mathrm{~K}$. This mechanism is different than those observed during compression of the perfect sphere and of the Wulff-like nanoparticle. This suggests that not only the contact surface area but also the edge geometries is a critical parameter for plastic deformation during compression.

\subsubsection{Cubic shape}

The analysis of simulations for cubic nanoparticles reveals two possible plastic deformation mechanisms. For all nanoparticles, except those of size greater or equal to $40 \mathrm{~nm}$ and with a)

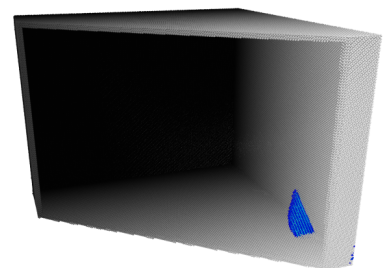

C)

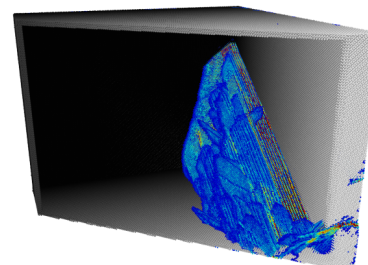

d)

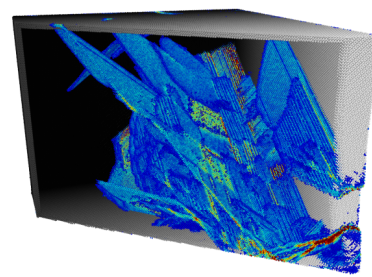

b)

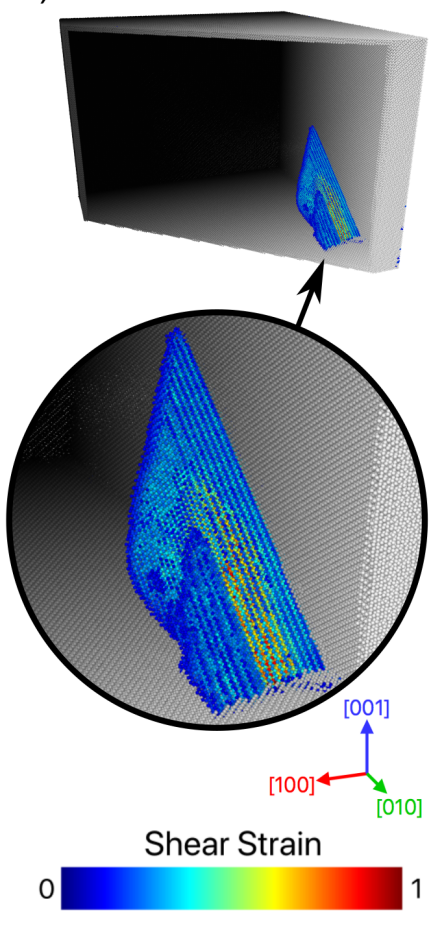

Figure 10: Onset and development of plastic deformation at $5 \mathrm{~K}$ in a $50 \mathrm{~nm}$ cubic silicon nanoparticle (perfect, $\alpha=0$ ). For clarity, perfect crystal atoms inside the nanoparticle are not shown, and the nanoparticle was cut. (a-d) von Mises shear strain visualization at true strains equal to 0.186 (a), 0.187 (b), 0.190 (c), 0.193 (d). An enlarged zone of the configuration (b) is also shown to better distinguish the plastic events.

$\alpha$ equal to 0.3 , plasticity occurs by the heterogeneous nucleation of a half-loop dislocation with a $\left(a_{0} / 2\right)<110>$ Burgers vector in a $\{110\}$ plane, from a contact surface (Fig. 10-a). This loop quickly expands, and is always accompanied with crossslip of the screw segment in a $\{111\}$ plane (Fig. 10-b). Subsequently, other cross-slip events in $\{111\}$ and $\{110\}$ planes can occur, as well as the repetition of the process from another surface sources (Fig. 10-c,d).

We observe a correlation between $\alpha$ and the relative participation of $\{110\}$ and $\{111\}$ planes at the onset of the plastic deformation. In fact, the lower $\alpha$ is, the lower the proportion of plastic deformation located in $\{111\}$ planes. In other words, the initial plastic deformation preferentially occurs in $\{111\}$ planes for blunt cubes, and in $\{110\}$ planes for sharp ones. The nanoparticle shape and the temperature also influence the location for the nucleation of the first dislocation. In most cases, the latter is found to form in the vicinity of cube corners, usually close to the edge of the contact surface. However, for sharp cubes $(\alpha=0-0.05)$ and at $300 \mathrm{~K}$, the first plastic events occurs from the lateral surfaces, and not in the corners.

A slightly different scenario is obtained for large cubic nanoparticles, with sizes greater or equal to $40 \mathrm{~nm}$, and with $\alpha=0.3$. In that case, the onset of plasticity corresponds to the heterogeneous nucleation of V-shaped $\{111\}$ half-loop dislocations from the corners (Fig. 11), similar to those obtained in Wulff-like and spherical nanoparticles. Unlike the previous 
a)

b)

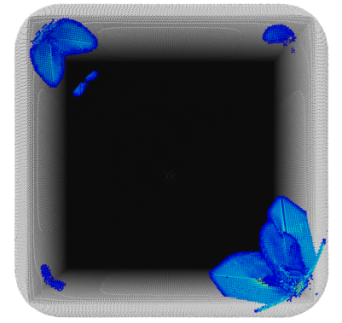

c)
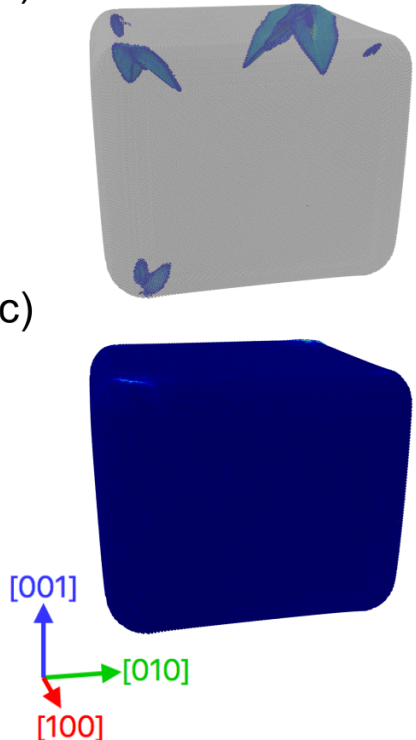
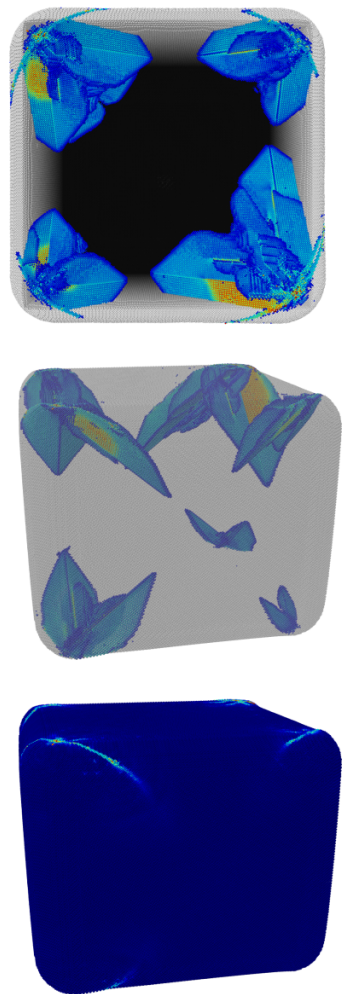
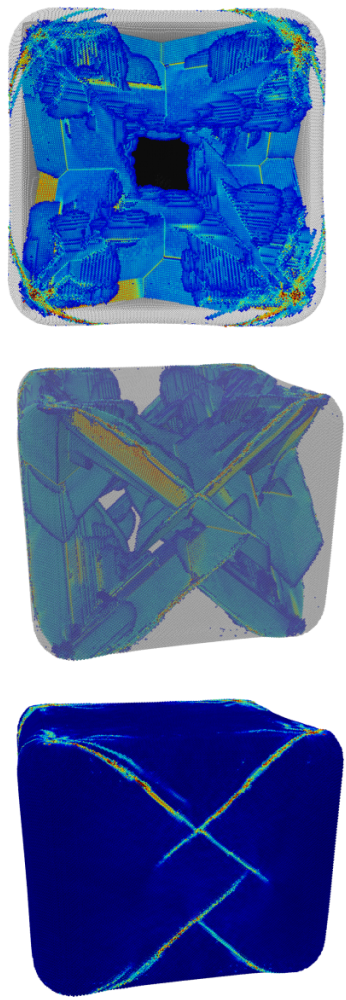

Shear Strain
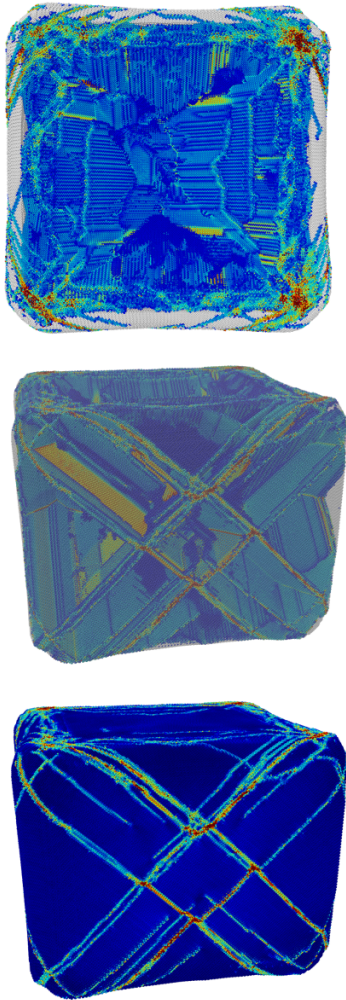

Figure 11: von Mises shear strain visualization revealing the onset and development of plastic deformation at $5 \mathrm{~K}$ in a $50 \mathrm{~nm}$ cubic silicon nanoparticle $(\alpha=0.30)$. a) Top view (along $<100>$ ), (b-c) side views showing or not the atoms in perfect crystal configuration inside the nanoparticle. The corresponding true strains are $0.164,0.165,0.166$, and 0.176 , from left to right.

process though, two different V-shaped dislocations are nucleated simultaneously at each corner. Thus all four possible $\{111\}$ slip planes are concerned. As deformation proceeds, loops expansion and new dislocation formations are observed, leading to a complex final structure (Fig. 11).

\section{Discussion}

A review of the literature on plastic deformation of silicon tells us that results and conclusions from molecular dynamics simulations can critically depend on the choice of interatomic potentials. This is obviously a severe issue, which may prevent emphatic claims from simulation-based investigations. Nevertheless, a thorough comparison of results obtained in the present work with recently reported studies brings interesting and encouraging agreements. First, an important point is that original mechanisms obtained here such as the V-shaped $\{111\}$ and $\{110\}$ dislocations were reported for other systems, and corroborated by electronic structure calculations [39, 57]. Furthermore, recent experimental studies of the mechanical properties of silicon nanowires and nanopillars revealed unexpected slip events located in $\{115\}$ planes $[41,42]$, which are also found in molecular dynamics simulations with the modified SW potential [42]. Also, in this last study, a comparison between experiments and simulations supports the dissociation process of a perfect shuffle $60^{\circ}$ dislocation into partial dislocations at low temperature, also observed in the present work. All these elements suggest that the potential used here is well suited for modeling most aspects of the plastic deformation of silicon. The only debating case would concern the nanosphere compressed along the $<100>$ orientation. In fact, some experiments first hinted that a phase transition, supposed to be $\beta$-tin, occurs below the indenter $[30,64]$. In these works, partial dislocations are next seemingly emitted from the metallic $\beta$-tin phase [30]. In our study, another plasticity mechanism is obtained, probably because the modified SW potential overestimates the $\beta$-tin phase transition pressure [48], thus favoring the homogeneous nucleation of dislocation loops at lower stresses. Therefore, the plasticity mechanism obtained in the present work in the specific case of the $<100>$ compression of spherical nanoparticles might not be realistic. Finally, it is also not clear whether the partial dislocations obtained in the case of the $<111>$ compression of both Wulff and spherical nanoparticles are the same than those observed by Wagner et al. for a $<100>$ compression [30]. In fact, the faulted areas in the simulations remain rather small, unlike in experiments.

Focusing now on the effect of size and shape, our results explicitly lead to the conclusion that the shape of the nanopar- 
ticles is a factor more influential than size for plasticity, or at least for the studied size range and silicon. In fact, depending on the shape, we observed both large yield stress variations between particles of similar size but with different shapes, together with diverse plasticity mechanisms. Conversely, the nanoparticle size has little influence on the plastic deformation mode in almost all cases. It is not clear whether such a conclusion could also apply for one-dimensional systems like nanopillars or nanowires. For instance, earlier investigations showed that the section geometry of silicon nanowires has little effect on plasticity mechanisms [65]. Conversely, it was demonstrated that the orientation of the compression greatly influences the plastic deformation mode of silicon nanopillars [40], a result also confirmed for nanoparticles in the present work.

In the present state of knowledge, it seems that no established and proven theories were put forward to explain the connection between plastic deformation and nanoparticle shape. The situation is indeed much more complicated compared to onedimensional systems. For the latter, although the strain state is not exactly spatially homogeneous due to the presence of lateral surfaces, it is often possible to only use simple concepts like Schmid factors to determine the resolved shear stresses for specific plasticity mechanisms [66]. Factors like cross section geometry were analyzed in several works, and it was shown that its main effect is to affect the critical length for nucleating dislocation half-loops $[67,68]$. In the case of nanoparticles, the compressive stress due to the indenter is not homogeneously distributed into the volume, except for a perfect nanocube which is a kind of idealized case. This is in part due to the fact that the indenter stress is transmitted through contact surfaces usually of lower dimensions than the nanoparticle. Also, the stress relaxation by lateral surfaces substantially depends on the shape. Finally, the nanoparticle geometry influences Poisson relaxation as well. It is for all these reasons that the strain/stress state in a compressed nanoparticle of arbitrary shape is typically complex and highly inhomogeneous.

The analysis of the stress tensor allows for understanding why specific plasticity mechanisms are obtained for a given nanoparticle shape. First, we focus on the V-shaped dislocations, nucleating in Wulff and spherically shaped nanoparticles. Maras and co-workers recently identified this mechanism as the possible primary step towards the formation of misfit dislocations in strained GeSi thin films [57]. The simultaneous dislocation nucleation in two different $\{111\}$ planes allows for an efficient relaxation of the bi-axial stress in these systems. In the present study, the large compression due to indenters also induces significant lateral stresses, which could explain the occurrence of these V-shaped dislocations. Next, we find that plastic deformation involved in several cases dislocations gliding in $\{110\}$ planes. This peculiar slip system was already reported in nanoparticles and nanowires for compression along $<100>[29,39,65]$. On the basis of electronic structure calculations, Guénolé et al. showed that a high stress component along $<100>$ favored the activation of the $\{110\}$ glide system, and not $\{111\}$ [39]. The uniaxial $<100>$ compression of our nanoparticles also yields large stresses along this orientation, which explains the occurence of $\{110\}$ dislocations. At last, the formation of the peculiar partial dislocations for $<111>$ compression is probably correlated to large and inhomogeneous stresses, although the exact relationship is difficult to extract. It is interesting that recently, $\mathrm{Li}$ and Picu showed that a suitable stress state could promote the dissociation of perfect shuffle into glide partial dislocations [69].

A simplified approach for analyzing the onset of plastic deformation in relation with the spatial distribution of stress is to examine the von Mises stress distribution. Figure 12 shows such views for different investigated systems, at the elastic limit, and confirms that the nanoparticle shape greatly influences the spatial distribution of stress. In particular, a strong inhomogeneity is revealed for the perfect spheres (Figure 12$\mathrm{a}, \mathrm{j})$. The presence of hemispherical stress fields for each contact surface is in agreement with predictions from the Hertz theory. Truncation of the sphere tends to reduce the inhomogeneity, and also to focus the stress maxima at the edges of contact surfaces (Figure 12-b,c,d). A similar pattern is obtained for Wulff-like nanoparticles (Figure 12-e,k). For the perfect cubic shape, we find a quasi homogeneous distribution of high stress values, with maxima located at the vicinity of the cube corners (Figure 12-f). Rounding the cube edges and corners makes the stress distribution less homogeneous, with the stress maxima following the edges of the contact surfaces (Figure 12$\mathrm{g}, \mathrm{h}, \mathrm{i})$. Finally, it is also worth noting that the influence of size on stress spatial distributions is negligible, for all nanoparticle shapes.

These spatial distributions also reveal a correlation between the localization of maximum stress values and the onset of plastic deformation in all cases. For instance, the homogeneous nucleation of a full dislocation loop for the perfect sphere is occuring in the high stress zones in Fig. 2-a, inside the nanoparticle. Also, all heterogeneous nucleations of half-loop dislocations are located at surface contact edges, where maximum stress values can be found. This suggests that it could be possible to guess where plastic deformation will be initiated in the nanoparticle by monitoring how stress is spatially building up during elastic compression. Such a proposition is likely to be softened depending on temperature, although we find a limited influence in the case of silicon.

The spatial distribution of stress and its evolution during the compression of the nanoparticle depend on size and shape. Actually it also depends on additional parameters such as the compression orientation, and eventually of the nature of the free lateral surfaces, although there is a clear relation between those and the nanoparticle shape. Our results indicate that shape has a more significant influence than size for plastic deformation. Unfortunately, the nanoparticle shape is too general as a parameter, since it can hardly be defined with a unique quantity, unlike size. Reviewing our results, the activated plasticity mechanisms depend primarily on the geometry of the contact surface, its orientation, as well as the ratio between the volume contained directly between the two contact surfaces and the nanoparticle volume. Thus we propose that these parameters should be focal points in future investigations of the mechanical properties of nanoparticles. 
a)

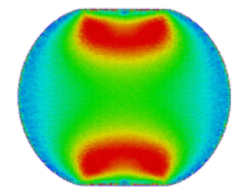

f)

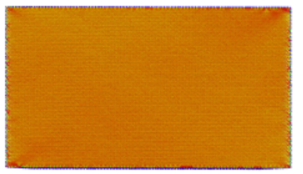

b)

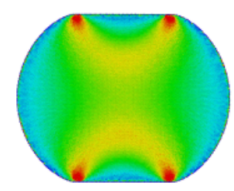

g)

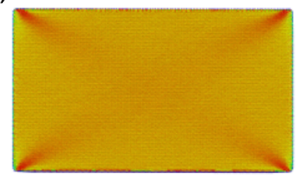

c)

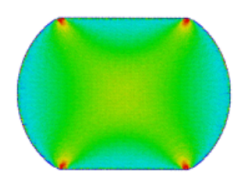

h)

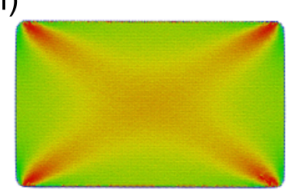

e)

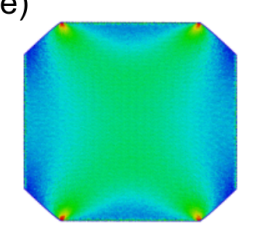

i)

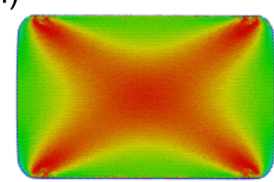

j)

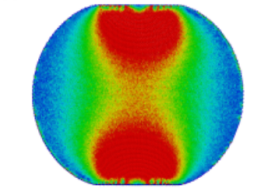

k)

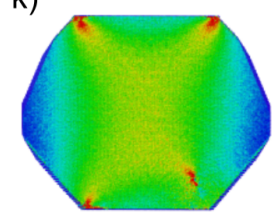

Figure 12: von Mises stress distributions at the elasticity limit, $5 \mathrm{~K}$ simulations, at the center of $50 \mathrm{~nm}$ nanoparticles: a) sphere b) T-sphere-20 c) T-sphere-40 d) T-sphere-80 e) Wulff-like f) cube (perfect) g) cube $(\alpha=0.05)$ h) cube $(\alpha=0.15)$ i) cube $(\alpha=0.3)$ j) sphere k) Wulff-like. a)-i) correspond to $<100>$ compressions, and j)-k) to $<111>$ compressions. For $<100>$, the cross-section plane is $\{011\}$ for the cubes, and $\{010\}$ for the other geometries. For $<111>$, the cross-section plane is $\{211\}$.

Table 2: Summary of plastic deformation mechanisms for each tested nanoparticle shape and compression orientation $(<100>$ when not mentioned). The number of $\star$ in the second column indicates qualitatively the magnitude of the size effect on yield stress (with $\bigcirc$ the absence of effect). Acronyms: Surface Nucleation (SN), Homogeneous Nucleation (HN), Cross-Slip (CS).

\begin{tabular}{|c|c|c|}
\hline System & Size effect & Plasticity mechanisms \\
\hline $\begin{array}{c}\text { Wulff } \\
<100>\end{array}$ & $\star \star \star$ & V-shaped $1 / 2<110>\{111\}$ shuffle $(\mathrm{SN})$ \\
\hline $\begin{array}{l}\text { Wulff } \\
<111>\end{array}$ & $\star \star \star$ & $\begin{array}{l}1 / 6<112>\{111\} \text { shuffle }(\mathrm{SN})+\mathrm{SF} \text { glide } \\
\rightarrow 1 / 2<110>\{111\} \text { shuffle }\end{array}$ \\
\hline $\begin{array}{l}\text { Sphere } \\
<100>\end{array}$ & $\bigcirc$ & $\begin{array}{l}1 / 2<110>\{111\} \text { shuffle }(\mathrm{HN}) \\
\rightarrow \text { V-shaped } 1 / 2<110>\{111\} \text { shuffle }(\mathrm{CS})\end{array}$ \\
\hline $\begin{array}{l}\text { Sphere } \\
<111>\end{array}$ & $\star$ & $\begin{array}{l}1 / 2<110>\{111\} \text { shuffle }(\mathrm{HN}) \\
\rightarrow 1 / 6<112>\{111\} \text { shuffle }+ \text { SF glide }\end{array}$ \\
\hline T-sphere & & $\begin{array}{l}1 / 2<110>\{111\} \text { shuffle }(\mathrm{SN}) \\
\rightarrow 1 / 2<110>\{110\}(\mathrm{CS})\end{array}$ \\
\hline $\begin{array}{l}\text { Cube } \\
\alpha=0\end{array}$ & $\bigcirc$ & $\begin{array}{l}1 / 2<110>\{110\}(\mathrm{SN}) \\
\rightarrow 1 / 2<110>\{111\} \text { shuffle }(\mathrm{CS})\end{array}$ \\
\hline $\begin{array}{c}\text { Cube } \\
\alpha=0.05 / 0.15\end{array}$ & $\star$ & $\begin{array}{l}1 / 2<110>\{110\}(\mathrm{SN}) \\
\rightarrow 1 / 2<110>\{111\} \text { shuffle }(\mathrm{CS})\end{array}$ \\
\hline $\begin{array}{c}\text { Cube } \\
\alpha=0.3\end{array}$ & $\star \star$ & $\begin{array}{l}\varnothing \leq 30 \mathrm{~nm}, \text { same as cubes with } \alpha \leq 0.15 \\
\varnothing \geq 40 \mathrm{~nm}, \text { V-shaped } 1 / 2<110>\{111\} \text { shuffle }(\mathrm{SN})\end{array}$ \\
\hline
\end{tabular}

\section{Conclusion}

In this work, we examine the mechanical response of silicon nanoparticles to an uniaxial compression by a flat-punch indenter, as a function of size and for different shapes, by performing large scale molecular dynamics calculations. The first main result is that no simple relation exists between the nanoparticle shape and the onset of plasticity. Furthermore, how mechanical properties are influenced by nanoparticle size also critically depends on the shape. For instance, no size effects are found for perfect cubic or spherical nanoparticles, compressed along $<100>$, but can be clearly identified for Wulff-shaped or blunt cubic systems (Table 2). The second main result is that a great variety of plasticity mechanisms is possible, and that which one is activated decisively depends on the nanoparticle shape (Table 2). In this work, we identify (i) the surface nucleation of a shuffle perfect dislocation half-loop in a single $\{111\}$ or double $\{111\}$ planes (V-shaped) (ii) the surface nucleation of a shuffle partial dislocation, leaving a glide stacking fault (iii) the surface nucleation of perfect dislocation in a $\{110\}$ plane (iv) the homogeneous nucleation of a shuffle perfect dislocation loop. Surface nucleation usually occurs at edges of a surface in contact with an indenter. We find that the activated plasticity mechanism is mainly determined by the location of the maximum local shear strain and by the geometry of the contact surfaces. Both features are obviously related to the nanoparticle shape.

Although our simulations and analyses only concern silicon, we are fairly confident that our conclusions could be also valid for other covalent materials. For metallic systems, one could also expect a strong influence of shape on mechanical properties, especially regarding the yield stress and the relation with size. These presumptions definitely call for future investigations.

\section{Acknowledgements}

This work was supported by ICFPAR/CEFIPRA (IndoFrench Centre for the Promotion of Advanced Research) in the framework of project 5208-1. Computations have been performed using the supercomputer facilities of the Meésocentre de calcul Poitou-Charentes, and HPC resources from GENCICINES (Grant 2017-A0030910302).

\section{References}

[1] O. Kraft, P. A. Gruber, R. Mönig, D. Weygand, Plasticity in confined dimensions, Annu. Rev. Mater. Res. 40 (1) (2010) 293-317. doi:10. 1146/annurev-matsci-082908-145409.

[2] J. R. Greer, J. T. D. Hosson, Plasticity in small-sized metallic systems: Intrinsic versus extrinsic size effect, Progress in Materials Science 56 (6) (2011) 654 - 724. doi:http: //dx.doi.org/10.1016/j.pmatsci.2011.01.005.

URL http://www.sciencedirect.com/science/article/pii/ S0079642511000065

[3] J. R. Greer, W. D. Nix, Nanoscale gold pillars strengthened through dislocation starvation, Phys. Rev. B 73 (2006) 245410. 
[4] W. D. Nix, S.-W. Lee, Micro-pillar plasticity controlled by dislocation nucleation at surfaces, Philos. Mag. 91 (7-9) (2011) 1084-1096. doi : 10.1080/14786435.2010.502141.

[5] X. W. Gu, Z. Wu, Y.-W. Zhang, D. J. Srolovitz, J. R. Greer, Microstructure versus flaw: Mechanisms of failure and strength in nanostructures, Nanoletters 13 (11) (2013) 5703-5709. doi : 10.1021/nl403453h. URL http: //dx.doi.org/10.1021/nl403453h

[6] S. Hoffmann, I. Utke, B. Moser, J. Michler, S. H. Christiansen, V. Schmidt, S. Senz, P. Werner, U. Gsele, C. Ballif, Measurement of the bending strength of vaporliquidsolid grown silicon nanowires, Nanoletters 6 (4) (2006) 622-625. doi : 10.1021/nl052223z.

[7] F. Östlund, K. Rzepiejewska-Malyska, K. Leifer, L. M. Hale, Y. Tang, R. Ballarini, W. W. Gerberich, J. Michler, Nanostructure fracturing: Brittle-to-ductile transition in uniaxial compression of silicon pillars at room temperature, Adv. Funct. Materials 19 (15) (2009) 2439-2444.

[8] F. Ureña, S. H. Olsen, L. Šiller, U. Bhaskar, T. Pardoen, J.-P. Raskin, Strain in silicon nanowire beams, J. Appl. Phys. 112 (11) (2012) 114506. doi:http://dx.doi.org/10.1063/1.4765025.

URL http://scitation.aip.org/content/aip/journal/jap/ 112/11/10.1063/1.4765025

[9] S. Lee, J. Im, Y. Yoo, E. Bitzek, D. Kiener, G. Richter, B. Kim, S. H. Oh, Reversible cyclic deformation mechanism of gold nanowires by twinningdetwinning transition evidenced from in situ tem, Nature Communications 5 (2014) 1.

URL http://dx.doi.org/10.1038/ncomms4033

10] B. N. Jaya, J. M. Wheeler, J. Wehrs, J. P. Best, R. Soler, J. Michler, C. Kirchlechner, G. Dehm, Microscale fracture behavior of single crystal silicon beams at elevated temperatures, Nanoletters 16 (12) (2016) 7597 7603. doi:10.1021/acs.nanolett.6b03461.

[11] M. D. Uchic, D. M. Dinmiduk, J. N. Florando, W. D. Nix, Sample dimensions influence strength and crystal plasticity, Science 305 (2004) 986.

[12] D. Guo, G. Xie, J. Luo, Mechanical properties of nanoparticles: basics and applications, J. Phys. D: Appl. Phys. 47 (1) (2014) 013001. URL http: //stacks.iop.org/0022-3727/47/i=1/a=013001

[13] M. T. McDowell, S. W. Lee, W. D. Nix, Y. Cui, 25th anniversary article: Understanding the lithiation of silicon and other alloying anodes for lithium-ion batteries, Adv. Mater. 25 (36) (2013) 4966-4985. doi : 10.1002/adma. 201301795 URL http://dx.doi.org/10.1002/adma. 201301795

[14] M. T. McDowell, S. W. Lee, J. T. Harris, B. A. Korgel, C. Wang, W. D. Nix, Y. Cui, In situ tem of two-phase lithiation of amorphous silicon nanospheres, Nanoletters 13 (2) (2013) 758-764.

[15] A. Pedersen, M. Bieri, M. Luisier, L. Pizzagalli, Lithiation of silicon nanoclusters, Phys. Rev. Applied 7 (2017) 054012. doi:10.1103/ PhysRevApplied.7.054012.

[16] X. H. Liu, L. Zhong, S. Huang, S. X. Mao, T. Zhu, J. Y. Huang, Sizedependent fracture of silicon nanoparticles during lithiation, ACS Nano 6 (2) (2012) 1522-1531. doi:10.1021/nn204476h.

[17] J. Deneen, W. Mook, A. Minor, W. Gerberich, C. Barry Carter, In situ deformation of silicon nanospheres, J. Mater. Sci. 41 (14) (2006) $4477-$ 4483.

[18] W. M. Mook, J. D. Nowak, C. R. Perrey, C. B. Carter, R. Mukherjee, S. L. Girshick, P. H. McMurry, W. W. Gerberich, Compressive stress effects on nanoparticle modulus and fracture, Phys. Rev. B 75 (2007) 214112 doi : 10.1103/PhysRevB.75.214112.

URL http://link.aps.org/doi/10.1103/PhysRevB.75.214112

[19] W. Gerberich, W. Mook, C. Perrey, C. Carter, M. Baskes, R. Mukherjee, A. Gidwani, J. Heberlein, P. McMurry, S. Girshick, Superhard silicon nanospheres, J. Mech. Phys. Solids 51 (6) (2003) 979 - 992. doi:http://dx.doi.org/10.1016/S0022-5096(03)00018-8. URL http://www.sciencedirect.com/science/article/pii/ S0022509603000188

[20] W. W. Gerberich, D. D. Stauffer, A. R. Beaber, N. I. Tymiak, A brittleness transition in silicon due to scale, J. Mater. Research 27 (2012) 552-561.

[21] X. Han, K. Zheng, Y. Zhang, X. Zhang, Z. Zhang, Z. L. Wang, Lowtemperature in situ large-strain plasticity of silicon nanowires, Adv. Mater. 19 (16) (2007) 2112.

[22] K. Kang, W. Cai, Brittle and ductile fracture of semiconductor nanowires - molecular dynamics simulations, Philos. Mag. 87 (14-15) (2007) 2169.

[23] D.-M. Tang, C.-L. Ren, M.-S. Wang, X. Wei, N. Kawamoto, C. Liu, Y. Bando, M. Mitome, N. Fukata, D. Golberg, Mechanical properties of si nanowires as revealed by in situ transmission electron microscopy and molecular dynamics simulations, Nanoletters 12 (2012) 1898.

[24] F. Abed El Nabi, J. Godet, S. Brochard, L. Pizzagalli, Onset of ductility and brittleness in silicon nanowires mediated by dislocation nucleation, Model. Simul. Mater. Sc. 23 (2) (2015) 025010.

URL http: //stacks . iop. org/0965-0393/23/i=2/a=025010

[25] K. Kendall, The impossibility of comminuting small particles by compression, Nature 272 (1978) 710.

[26] A. Beaber, J. Nowak, O. Ugurlu, W. Mook, S. Girshick, R. Ballarini, W. Gerberich, Smaller is tougher, Philos. Mag. 91 (79) (2011) 1179-1189. arXiv:http://dx.doi.org/10.1080/ 14786435.2010.487474, doi:10.1080/14786435.2010.487474. URL http://dx.doi.org/10.1080/14786435.2010.487474

[27] K.-C. Fang, C.-I. Weng, S.-P. Ju, An investigation into the mechanical properties of silicon nanoparticles using molecular dynamics simulations with parallel computing, J. Nanopart. Res. 11 (3) (2009) 581-588. doi : $10.1007 / \mathrm{s} 11051-008-9396-\mathrm{x}$ URL http://dx.doi.org/10.1007/s11051-008-9396-x

[28] N. Zhang, Q. Deng, Y. Hong, L. Xiong, S. Li, M. Strasberg, W. Yin, Y. Zou, C. R. Taylor, G. Sawyer, Y. Chen, Deformation mechanisms in silicon nanoparticles, J. Appl. Phys. 109 (6) (2011) 063534. doi:http://dx.doi.org/10.1063/1.3552985.

URL http://scitation.aip.org/content/aip/journal/jap/ 109/6/10.1063/1.3552985

[29] L. Hale, D.-B. Zhang, X. Zhou, J. Zimmerman, N. Moody, T. Dumitrica, R. Ballarini, W. Gerberich, Dislocation morphology and nucleation within compressed si nanospheres: A molecular dynamics study, Comp. Mat. Sci. 54 (0) (2012) 280 - 286. doi:http://dx.doi.org/10.1016/j.commatsci.2011.11.004. URL http://www.sciencedirect.com/science/article/pii/ S0927025611006185

[30] A. J. Wagner, E. D. Hintsala, P. Kumar, W. W. Gerberich, K. A. Mkhoyan, Mechanisms of plasticity in near-theoretical strength sub-100 nm si nanocubes, Acta Mater. 100 (2015) 256 - 265. doi:https://doi.org/10.1016/j.actamat.2015.08.029.

URL http://www.sciencedirect.com/science/article/pii/ $\mathrm{S} 1359645415006060$

[31] L. Yang, J. J. Bian, H. Zhang, X. R. Niu, G. F. Wang, Size-dependent deformation mechanisms in hollow silicon nanoparticles, AIP Advances 5 (7) (2015) 077162. doi:http://dx.doi.org/10.1063/1. 4927509

URL http://scitation.aip.org/content/aip/journal/adva/ 5/7/10.1063/1.4927509

[32] P. Valentini, W. W. Gerberich, T. Dumitrică, Phase-transition plasticity response in uniaxially compressed silicon nanospheres, Phys. Rev. Lett. 99 (2007) 175701. doi:10.1103/PhysRevLett.99.175701.

URL http://link.aps.org/doi/10.1103/PhysRevLett.99. 175701

[33] L. Hale, X. Zhou, J. Zimmerman, N. Moody, R. Ballarini, W. Gerberich, Phase transformations, dislocations and hardening behavior in uniaxially compressed silicon nanospheres, Comp. Mat. Sci. 50 (5) (2011) 1651 1660. doi : http://dx.doi.org/10.1016/j.commatsci.2010.12. 023.

URL http://www.sciencedirect.com/science/article/pii/ S0927025610006944

[34] Y. Hong, N. Zhang, M. A. Zaeem, Metastable phase transformation and deformation twinning induced hardening-stiffening mechanism in compression of silicon nanoparticles, Acta Mater. 145 (2018) 8 - 18 . doi:https://doi.org/10.1016/j.actamat.2017.11.034. URL https://www.sciencedirect.com/science/article/pii/ S1359645417309771

[35] J. Amodeo, K. Lizoul, Mechanical properties and dislocation nucleation in nanocrystals with blunt edges, Materials \& Design 135 (2017) 223 231. doi:https://doi.org/10.1016/j.matdes.2017.09.009.

[36] A. S. Barnard, P. Zapol, A model for the phase stability of arbitrary nanoparticles as a function of size and shape, J. Chem. Phys. 121 (9) (2004) 4276-4283. doi : 10.1063/1.1775770.

[37] J. D. Nowak, C. B. Carter, Morphology of semiconductor nanoparticles, in: A. G. Cullis, P. A. Midgley (Eds.), Microscopy of Semiconducting Materials 2007, Springer Netherlands, Dordrecht, 2008, pp. 285-288.

[38] J. Rabier, L. Pizzagalli, J.-L. Demenet, Dislocations in silicon at high 
stress, in: L. Kubin, J. P. Hirth (Eds.), Dislocation in solids, Vol. 16, Elsevier, 2010, Ch. 93, p. 47.

[39] J. Guénolé, S. Brochard, J. Godet, Unexpected slip mechanism induced by the reduced dimensions in silicon nanostructures: Atomistic study, Acta Mater. 59 (2011) 7464.

[40] Y. He, L. Zhong, F. Fan, C. Wang, T. Zhu, S. X. Mao, In situ observation of shear-driven amorphization in silicon crystals, Nature Nanotechnology 11 (10) (2016) 866-871. doi :10.1038/nnano.2016.166.

[41] L. Vincent, D. Djomani, M. Fakfakh, C. Renard, B. Belier, D. Bouchier, G. Patriarche, Shear-driven phase transformation in silicon nanowires, Nanotechnology 29 (12) (2018) 125601. doi:10.1088/1361-6528/ aaa738.

[42] M. Texier et al., in preparation (2018).

[43] A. Stekolnikov, J. Furthmüller, F. Bechstedt, Absolute surface energies of group-iv semiconductors: Dependence on orientation and reconstruction, Phys. Rev. B 65 (2002) 115318.

[44] F. H. Stillinger, T. A. Weber, Computer simulation of local order in condensed phases of silicon, Phys. Rev. B 31 (8) (1985) 5262.

[45] J. Tersoff, Modeling solid-state chemistry: Interatomic potentials for multicomponent systems, Phys. Rev. B 39 (8) (1989) 5566.

[46] J. Godet, L. Pizzagalli, S. Brochard, P. Beauchamp, Comparison between classical potentials and ab initio methods for silicon under large shear, J. Phys.: Condens. Matter 15 (2003) 6943.

[47] J. Godet, P. Hirel, S. Brochard, L. Pizzagalli, Evidence of two plastic regimes controlled by dislocation nucleation in silicon nanostructures, $\mathrm{J}$. Appl. Phys. 105 (2009) 026104.

[48] L. Pizzagalli, J. Godet, J. Guénolé, S. Brochard, E. Holmstrom, K. Nordlund, T. Albaret, A new parametrization of the stillingerweber potential for an improved description of defects and plasticity of silicon, Journal of Physics: Condensed Matter 25 (5) (2013) 055801.

[49] S. Plimpton, Fast parallel algorithms for short-range molecular dynamics, J. Comput. Phys. 117 (1) (1995) $1-19$.

[50] Http://lammps.sandia.gov/.

[51] T. D. B. Jacobs, A. Martini, Measuring and understanding contact area at the nanoscale: A review, Applied Mechanics Reviews 69 (6) (2017) 060802 URL http://dx.doi.org/10.1115/1.4038130

[52] M. Vergeles, A. Maritan, J. Koplik, J. R. Banavar, Adhesion of solids, Phys. Rev. E 56 (3) (1997) 2626-2634. doi : 10.1103/PhysRevE. 56. 2626.

[53] A. Stukowski, Visualization and analysis of atomistic simulation data with ovito-the open visualization tool, Modelling and Simulation in Materials Science and Engineering 18 (1) (2010) 015012.

[54] Http://ovito.org/.

[55] Y. Feruz, D. Mordehai, Towards a universal size-dependent strength of face-centered cubic nanoparticles, Acta Mater. 103 (2016) 433 - 441 doi:http://dx.doi.org/10.1016/j.actamat.2015.10.027. URL http://www.sciencedirect.com/science/article/pii/ S1359645415300276

[56] D. Chrobak, N. Tymiak, A. Beaber, O. Ugurlu, W. Gerberich, R. Nowak, Deconfinement leads to changes in the nanoscale plasticity of silicon, Nature Nanotechnology 6 (2011) 480.

[57] E. Maras, L. Pizzagalli, T. Ala-Nissila, H. Jónsson, Atomic scale formation mechanism of edge dislocation relieving lattice strain in a gesi overlayer on si(001), Scientific Reports 7 (1) (2017) 11966. doi:10.1038/ s41598-017-12009-y.

[58] L. Pizzagalli, J. Godet, S. Brochard, Glissile dislocations with transient cores in silicon, Phys. Rev. Lett. 103 (2009) 065505.

[59] J. Hornstra, Dislocations in the diamond lattice, J. Phys. Chem. Solids 5 (1958) 129

[60] L. Pizzagalli, J. Godet, S. Brochard, Influence of strain on dislocation core in silicon, Philos. Mag. 98 (13) (2018) 1151-1169. doi:10.1080/ 14786435.2018.1431412.

[61] D. Rodney, L. Ventelon, E. Clouet, L. Pizzagalli, F. Willaime, Ab initio modeling of dislocation core properties in metals and semiconductors, Acta Mater. 124 (2017) 633-659. doi:10.1016/j.actamat. 2016. 09.049.

[62] F. Louchet, J. Thibault-Desseaux, Dislocation cores in semiconductors. from the shuffle or glide dispute to the glide and shuffle partnership, Revue de Physique Appliquée 22 (1987) 207.

[63] J. Godet, L. Pizzagalli, S. Brochard, P. Beauchamp, Computer study of microtwins forming from surface steps of silicon, Comp. Mat. Sci. 30 (2004) 16.

[64] W. W. Gerberich, W. M. Mook, M. J. Cordill, C. B. Carter, C. R. Perrey, J. V. Heberlein, S. L. Girshick, Reverse plasticity in single crystal silicon nanospheres, Int. J. of Plasticity 21 (12) (2005) 2391 - 2405. doi:http://dx.doi.org/10.1016/j.ijplas.2005.03.001. URL http://www.sciencedirect.com/science/article/pii/ S0749641905000550

[65] Z. Yang, Z. Lu, Y.-P. Zhao, Shape effects on the yield stress and deformation of silicon nanowires: A molecular dynamics simulation, J. Appl. Phys. 106 (2) (2009) 023537. doi:10.1063/1.3186619.

[66] C. R. Weinberger, W. Cai, Plasticity of metal nanowires, J. Materials Chemistry 22 (8) (2012) 3277. doi : 10.1039/c2jm13682a.

[67] T. Zhu, J. Li, A. Samanta, A. Leach, K. Gall, Temperature and strain-rate dependence of surface dislocation nucleation, Phys. Rev. Lett. 100 (2008) 025502 .

[68] C. R. Weinberger, A. T. Jennings, K. Kang, J. R. Greer, Atomistic simulations and continuum modeling of dislocation nucleation and strength in gold nanowires, J. Mech. Phys. Solids 60 (1) (2012) 84 - 103. doi : https ://doi.org/10.1016/j.jmps . 2011.09.010.

[69] Z. Li, R. Picu, Shuffle-glide dislocation transformation in si, J. Appl. Phys. 113 (2013) 083519 